\title{
Development of Retinal Displaced Ganglion Cells in the Chick: Neurogenesis and Morphogenesis
}

\author{
Carmen Prada, ${ }^{1}$ Juan I. Medina, ${ }^{1}$ Rosario López, ${ }^{1}$ José M. Génis-Gálvez, ${ }^{2}$ and Francisco A. Prada ${ }^{2}$ \\ 'Departamento de Fisiologia, Facultad de Medicina, Universidad Complutense, 28040 Madrid, Spain and 2 Departamento \\ de Ciencias Morfológicas, Facultad de Medicina, Universidad de Sevilla, 41009 Sevilla, Spain
}

\begin{abstract}
The time of birth of displaced ganglion cells (DGCs) was determined by autoradiography. DGCs start to leave the cell cycle early, on embryonic day 3 , in the central and peripheral retina, and end on embryonic day 8 , also in both areas of the retina. During the period of neurogenesis, unlabeled (born) DGCs do not appear distributed in spatial gradients as do the ganglion, amacrine, and other cell types in the retina (Prada et al., 1991). Our results show characteristic spatial and temporal patterns of DGC neurogenesis, which differ from those of the other retinal cell types.

The morphogenesis of DGCs was studied by means of Golgi preparations. After leaving the cycle, DGC neuroblasts detach from the ventricular lining; they then move their soma through the vitreal process toward the final position at the same time that they emit the axon. Also during soma translocation, a transient sprouting of a few short processes is emitted from the vitreal process of the cell, close to where the soma is later located, suggesting that the "abnormal" position of DGCs could be speciflcally marked during the process of migration.
\end{abstract}

In the vertebrate retina, the displaced ganglion cells (DGCs) constitute a relatively small population of cells, $2 \%$ of the total number of ganglion cells in the mammalian retina (mouse, Dräger and Olsen, 1981), and $0.43 \%$ in the avian retina (chick, Prada et al., 1989). Their cell bodies are scattered along the inner margin of the inner nuclear layer (inl), mixed with amacrine cell bodies. The axon of DGCs descends to the layer of optic axons, traversing the inner plexiform layer (ipl) and the ganglion cell layer (gcl), to the visual centers in the brain. DGCs have been described in all vertebrate classes, including primates (Boycott and Dowling, 1969; Bunt and Minckler, 1977).

Since the early studies by Dogiel (1888) and Ramón y Cajal (1933), DGCs have generally been regarded as a population of ganglion cells anomalously located. Consequently, a theory has emerged that postulates some ontogenetic "accident" inhibiting the migration of these neurons toward the gcl (Boycott and Dowling, 1969; Bunt and Minckler, 1977; Maslim et al., 1986; Linden, 1987; Buhl and Dann, 1988). Some of these authors (Bunt and Minckler, 1977; Dräger and Olsen, 1980; Linden,

\footnotetext{
Received Oct. 25, 1991; revised Feb. 21, 1992; accepted Apr. 14, 1992.

This work was supported by the Dirección General de Investigación Científica y Técnica (Grant PS 87-0033) and Fondo de Investigaciones Sanitarias de la Seguridad Social (Grant 88/1684).

Correspondence should be addressed to Dra. Carmen Prada, Departamento de Fisiología, Facultad de Medicina, Universidad Complutense, Ciudad I Iniversitaria s/n, 28040 Madrid, Spain.

Copyright (C) 1992 Society for Neuroscience $0270-6474 / 92 / 123781-08 \$ 05.00 / 0$
}

1987) reasonably proposed a late neurogenesis for DGCs, as the determinant of their failure in the migratory process. According to this proposition, DGCs would be the last ganglion cells to be generated, their neurogenesis coinciding with the last part of the neurogenesis period of amacrine cells, which would explain their presence among the latter. The present study (the first on the development of DGCs) shows that DGCs start to be generated very early during development, and do not follow the spatial and temporal patterns of neurogenesis of either ganglion or amacrine cells (Prada et al., 1991). In addition, we have been able tentatively to identify, by means of the Golgi method, the earlicst DGC neuroblasts (i.e., migrating neuroblasts) and have found a morphogenetic sequence by which they would be transformed into mature cells.

\section{Materials and Methods \\ Tissue section autoradiographs}

White Leghorn chick embryos were raised to the desired stage of development in a forced-draft incubator, at $37.5^{\circ} \mathrm{C}$ and $60 \%$ relative humidity. Fertilized chicken eggs were obtained from poultry of the Center of Molecular Biology at Autónoma University. Two embryos were injected daily between 2 and $11 \mathrm{~d}$ of incubation (E2-E11). Each embryo received a single dose of $25 \mu \mathrm{Ci}$ of $6-{ }^{3} \mathrm{H}$-thymidine (Amersham; $23 \mathrm{Ci}$ / $\mathrm{mmol}$, at $1 \mathrm{mCi} / \mathrm{ml}$ ) into the chorioallantois, through a window made in the shell. All embryos were killed at E14, when the retina is already organized in layers. Pieces containing both eyes were cut from the heads with a razor blade and fixed in Bouin's solution. Then the tissues were rinsed in water, immersed in ammoniated $70 \%$ alcohol to eliminate any residual picric acid, and finally embedded in paraffin. Transversal serial sections (parallel to $\mathrm{YY}^{\prime}$; Fig. 1) of $6-10 \mu \mathrm{m}$ were cut, mounted on albumin-coated slides, and processed for conventional autoradiography. Kodak NTB2 emulsion, diluted 1:1 with distilled water, was used in this process. The autoradiographs were exposed for $3-4$ weeks at $4^{\circ} \mathrm{C}$, and after development with Kodak D-19 they were "weakly" stained with $1 \%$ thionin, dehydrated, and coverslipped.

\section{Evaluation of autoradiographs}

Identification of DGCs. DGCs appear particularly prominent in the chick retina when paraffin sections are stained weakly with thionin. Their cell bodies are strongly stained, showing dark cytoplasm and a large clear nucleus (see Fig. 2 in Results). They are large, 18-30 $\mu \mathrm{m}$ at the largest diameter, which is three to four times the average size of other retinal cell types; they are located with amacrine cells in the inner border of the inl, are widely spaced (rarely closer than $100 \mu \mathrm{m}$ ), and arc morc numerous in the peripheral than in the central retina. All these characteristics have been recognized as typical of DGCs in the chick retina (Reiner et al., 1979; Prada et al., 1989), and no type of amacrine cell located with them in the inner row of the inl exhibits all these characteristics (see Discussion).

Quantitative evaluations. As mentioned above, in our experiments 25 $\mu \mathrm{Ci}$ of ${ }^{3} \mathrm{H}$-thymidine was introduced into each embryo, which is sufficient to ensure that every neuroepithelial cell entering the cycle would incorporate the precursor into the DNA. The progeny of these cells would then appear labeled in autoradiographs prepared at some later 


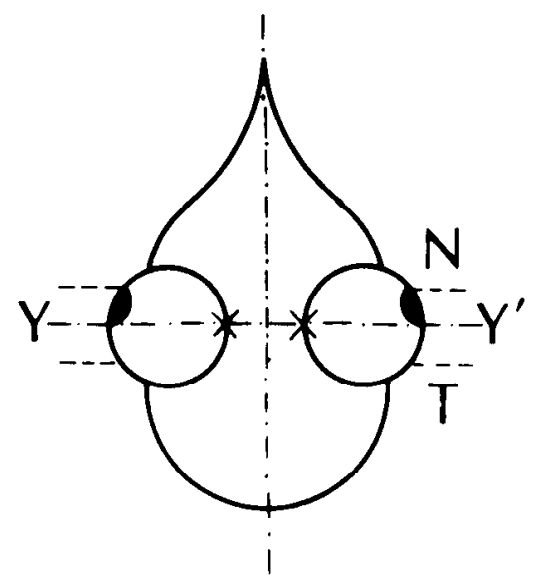

Figure 1. Top (dorsal) view of a chick head showing the position of the transversal plane $Y Y^{\prime}$, which contains both retinal posterior poles (crosses), and separates the nasal $(N)$ and temporal $(T)$ halves. The sections used in this study are parallel to this plane, which is also referred to in the text as the median plane. The other broken parallel lines indicate the approximate nasal and temporal levels of sections in which quantitative evaluations were made.

stage of development. Any cells that had left the cycle up to the time of injection would, however, appear unlabeled. Therefore, we considered as born cells the unlabeled ones.

Chick retina contains a total population of approximately 10,400 DGCs (Prada et al., 1989), so only a mean of 16 cells with a well delimited nucleus can be found in a single section passing through the lens and the posterior pole of the retina (Fig. 1, YY'), at which level the length of retinal sections is greatest. Therefore, a very small number of cells is found in each one of the retinal sectors (dorsoperipheral, dorsoequatorial, dorsocentral, ventrocentral, and ventroperipheral), considered in a previous study by Prada et al. (1991), which requires the evaluation of a large number of sections for a trustworthy comparison among sectors. Thus, because DGCs are not homogeneously distributed throughout the retina, and because in contrast with other retinal cell types they are more numerous in the peripheral than in the central retina, we did not use the same method of analysis employed recently to study the spatial and temporal patterns of neurogenesis of the other retinal cell types (Prada et al., 1991). In this study, we considered a single central sector in each retinal section and two peripheral sectors, the dorsoperipheral and the ventroperipheral, as indicated in Figure 4. Labeled and unlabeled DGCs were separately counted in the different sectors by scanning each section. Twenty consecutive sections passing

Table 1. Number of embryos of each stage, stained by the Golgi method, used in this study

\begin{tabular}{|c|c|c|}
\hline $\begin{array}{l}\text { Stage } \\
\text { (Hamburger } \\
\text { and Hamilton, } \\
1951 \text { ) }\end{array}$ & Embryonic age (d) & $\begin{array}{l}\text { Number of } \\
\text { embryos studied }\end{array}$ \\
\hline 31 & 7 & 10 \\
\hline 34 & 8 & 7 \\
\hline 35 & 9 & 11 \\
\hline 36 & 10 & 9 \\
\hline 37 & 11 & 9 \\
\hline 38 & 12 & 9 \\
\hline 40 & 14 & 11 \\
\hline 41 & 15 & 5 \\
\hline 42 & 16 & 7 \\
\hline 44 & 18 & 5 \\
\hline 46 & 20 & 6 \\
\hline Total & & 89 \\
\hline
\end{tabular}

Table 2. Percentages of born DGCs found in DP, $C$, and VP sectors of sections at the nasal, median, and temporal levels, when the injection of ${ }^{3} \mathrm{H}$-thymidine was given at $\mathbf{E 4}$

\begin{tabular}{lccc} 
& DP & C & VP \\
\hline Nasal & 6.2 & 5 & 1.2 \\
Median & 18.4 & 13 & 4.2 \\
Temporal & 18.7 & 14.2 & 6.9 \\
\hline
\end{tabular}

at each one of the nasal, median, and temporal levels (Fig. 1) were evaluated to obtain the percentage of unlabeled cells in each sector. The autoradiographs of retinas of the two embryos injected per stage were indistinguishable, and thus the evaluations were made in either.

\section{Golgi preparations}

Because of the small number of DGCs in the retina, and since the Golgi method stains only $10 \%$ of the cells in good impregnations, a large number of embryos of each stage had to be processed to ensure the staining of DGC ncuroblasts at all stages. Table 1 shows the number of embryos of each stage, from E7 to E20, that were successfully stained. Embryos from E7 to E14 were decapitated, and the heads were immersed in Stensaas (1967) solution for fixation for 3-4 d. Embryos from E14 to E20 were enucleated and the eye globes immersed in Colonnier (1964) fixative for several days (up to 2 weeks). After washing in tap water, the tissues were stained with silver nitrate, dehydrated, and embedded in low-viscosity nitrocellulose. Serial sections, 70-100 $\mu \mathrm{m}$ thick, were made also in the transversal plane and mounted with Dammar resin following the procedure described by Prada and López-Mascaraque (1985).

\section{Results}

\section{Neurogenesis}

DGCs start to leave the cycle throughout all the retina at E3; the autoradiographs of retinas injected at E4 show a small number of unlabeled DGCs in all sectors and at any retinal level of cut (Table 2). DGCs stop leaving the cycle at E8, since the autoradiograms of retinas labeled at E9 show $100 \%$ of unlabeled DGCs throughout the retina, so DGCs are produced from E3 to E9. This period of neurogenesis, although the shortest, is within the period of neurogenesis of ganglion cells, from E2 to E9, and amacrine cells, from E3 to E10 (Prada et al., i991). This indicates that DGCs are not the last ganglion cells to leave the cycle and also that they stop leaving the cycle before some amacrine cells. Examples of DGCs born before some ganglion and amacrine cells are shown in Figure $2 c-h$.

To study the spatial and temporal patterns of DGC neurogenesis, the distribution of unlabeled cells in the different sectors, central (C), dorsoperipheral (DP), and ventroperipheral (VP) of sections passing at the nasal, median, and temporal levels of the retina, was analyzed at each stage of injection (autoradiograms of some DGCs counted in the different sectors are shown in Fig. 2). Table 2 shows the results obtained from retinas labeled at $\mathrm{E} 4$, when $\mathrm{DGC}$ neurogenesis starts. The DP sector has a slightly higher number of unlabeled cells than the $\mathrm{C}$ sector, and both sectors have many more unlabeled cells than the VP one. This initial advantage in DGC neurogenesis of the dorsal retina over the ventral is maintained throughout the period of neurogenesis, as can be appreciated in Figure 3. Table 2 also indicates that each sector contains very similar numbers of unlabeled cells at the median and temporal levels, these numbers being much higher than those at the nasal level. Therefore, the temporal retina starts to generate more unlabeled cells than the nasal one, and this difference is maintained throughout neurogenesis. 

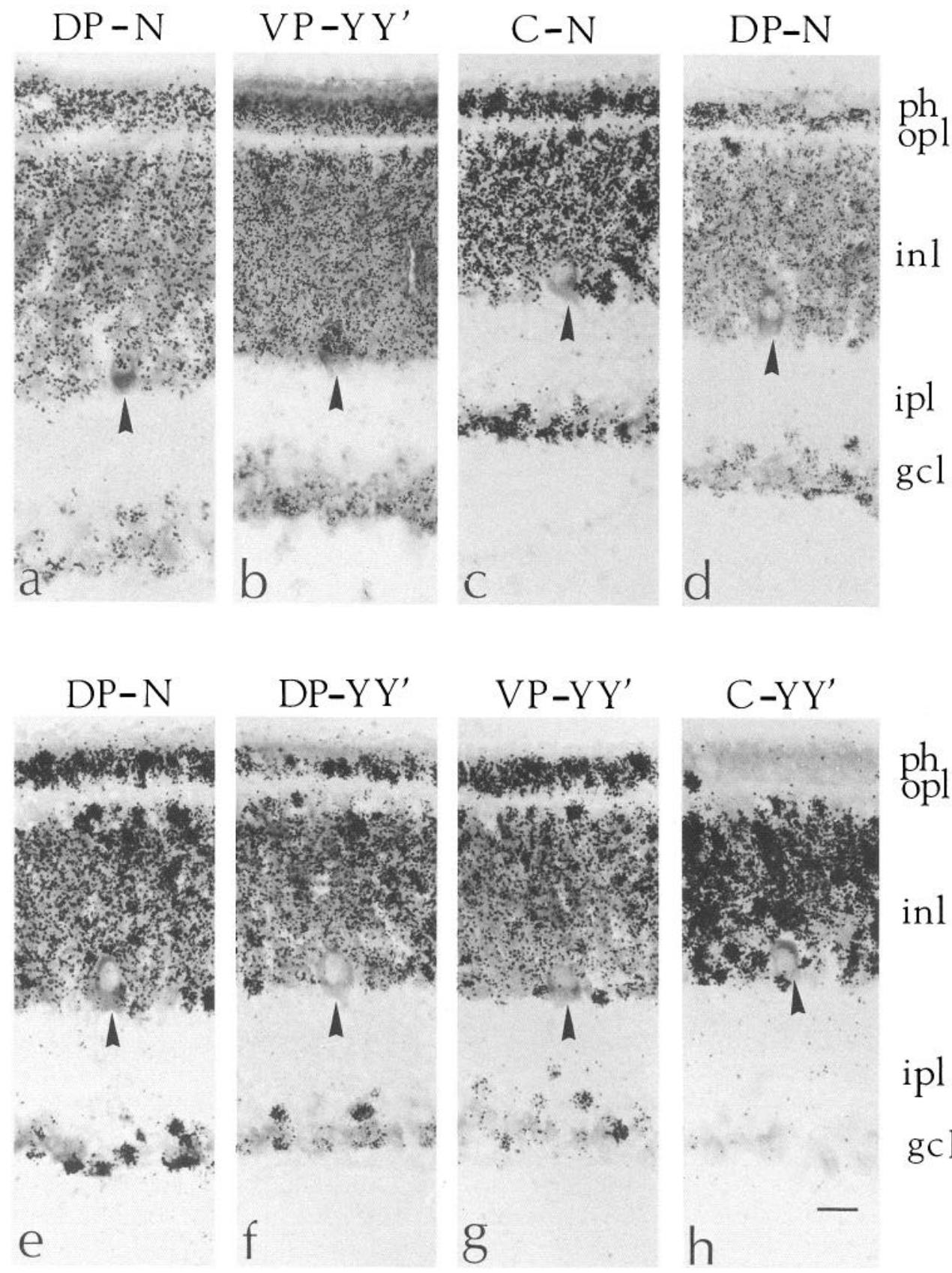

The small difference in the percentages of unlabeled DGCs between the DP and C sectors of retinas labeled at E4 disappears at the following stages of development. Both sectors have the same number of born DGCs from E5 onward (Fig. 3). These results were unexpected because the other retinal cell types, in the chick (Kahn, 1973, 1974; Prada et al., 1991) as well as in other species studied, are produced in a central-to-peripheral gradient, and therefore more unlabeled cells should have been found in the central sector than in the peripheral sectors at any stage of neurogenesis. However, our results show that the DP sector contains proportionally more unlabeled cells than the $\mathrm{C}$ sector, since although both sectors contain the same number of unlabeled cells, the DP sector is smaller than the C sector (see the lengths of sectors in Fig. 4). Therefore, these results do not support the existence of a central-to-peripheral gradient of DGC neurogenesis.

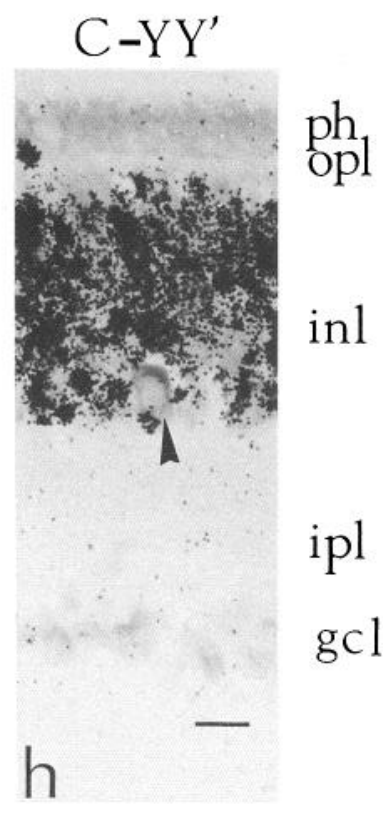

Figure 2. Section autoradiograms of retinas labeled at $\mathrm{E} 4(a, b), \mathrm{E} 6(c, d)$, and E7 $(e-h)$, to show examples of labeled and unlabeled DGCs (arrowheads) in different retinal sectors. At the top of each autoradiogram is indicated the sector $(D P$, dorsoperipheral; $V P$, ventroperipheral; $C$, central) and level of cut $\left(N\right.$, nasal; $Y Y^{\prime}$, median; sections at the temporal level are not shown) of the corresponding section. ph, photoreceptor layer; opl, outer plexiform layer; inl, inner nuclear layer; ipl, inner plexiform layer; $g c l$, ganglion cell layer. Note that the large size of the soma and nucleus of the DGCs makes it easy to discern labeled and unlabeled cells. Even in the peripheral sectors of retinas labeled at early stages of development (up to E6), where the label is homogeneously distributed and the nucleus of the other retinal cell types contains only a few grains, the identification of labeled $(a$ and $b$ ) and unlabeled $(d)$ cells does not offer any doubt. Autoradiograms in $c-g$ clearly show that some DGCs are born before many ganglion cells of the same sector. The autoradiogram in $h$ shows that some DGCs are born before most amacrine cells (the inner third of the inl) in the same sector. Scale bar, $20 \mu \mathrm{m}$.

When analyzing the sections individually, neither the centralto-peripheral gradient nor other spatial gradients are apparent, as occurs in the case of the other retinal cell types that show central-to-peripheral, dorsal-to-ventral, and temporal-to-nasal gradients (Prada et al., 1991). In Figure 4, we indicate the real position of all (labeled and unlabeled) DGCs found in sections passing at the same level (through the lens and the posterior pole) of retinas injected at different stages of neurogenesis. The section in Figure $4 a$, from a retina labeled at E4, shows only one unlabeled cell in the DP sector. Other sections close to this in the series contained only one unlabeled cell in the VP sector or one unlabeled cell in the $\mathrm{C}$ sector. Some sections did not contain any unlabeled cells; others contained one unlabeled cell per sector in the three sectors, while others showed one unlabeled cell per sector in two sectors (either DP and VP, or DP and $\mathrm{C}$, or C and VP). Two or three unlabeled cells per sector 


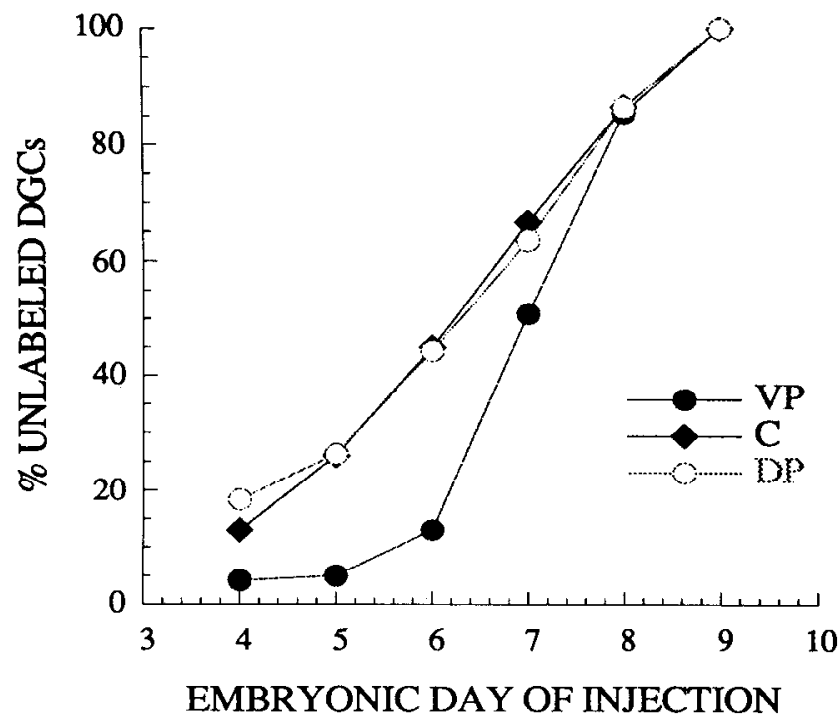

Figure 3. The percentage of born (unlabeled) DGCs in each retinal sector ( $D P$, dorsoperipheral; $C$, central; $V P$, ventroperipheral) is plotted as a function of the incubation time at which ${ }^{3} \mathrm{H}$-thymidine was injected. Each point was obtained evaluating 20 consecutive median sections per day of incubation.

were also found. Therefore, these results show that at the beginning of neurogenesis, central-to-peripheral and dorsal-toventral gradients are not apparent. Neither are they at the following stages of neurogenesis, in which we found the same heterogeneity in the distribution of unlabeled cells. Examples of sections from retinas labeled at E6 and E7 are presented in Figure $4, b$ and $c$. The analysis of sections at the nasal and temporal levels did not reveal any gradient (examples of these are not presented).

The time course of accumulation of born DGCs is shown in Figure 3. The values were obtained in sections at the median level, that is, through YY' (Fig. 1). In the DP and C sectors, the rate of production is fairly constant during the period of neurogenesis; $18 \%$ of cells (on an average) are produced at each stage. However, in the VP sector the rate of production varies with the stage; it is very low at E4 and E5 (around 4\%), high at E6 and E7 (38\% and 35\%, respectively), and low at E8 (around $14 \%$ ). The analysis of nasal and temporal sections gave similar profiles. The profile obtained for the DP and C sectors is different from that found for the other retinal cell types (Prada et al., 1991), whose rates of production change with the stage, and furthermore all of them have a peak of production during E6 in the dorsocentral sector, not shown by DGCs. This is not the only difference in the temporal pattern of neurogenesis between DGCs and the other retinal cells. As already said, DGCs start to leave the cycle throughout the retina, while the other cells studied do so only in a defined dorsotemporal area (Prada et al., 1991). One hundred percent of born DGCs are found in all retinal sectors at the same time, E9 (Fig. 3), while $100 \%$ of unlabeled cells of the other types are found first in the dorsocentral sector of temporal sections, then extending from this sector to the dorsoperipheral and then to the ventral sectors, and at the same time extending from the temporal area to the nasal one, in the same manner that the beginning of neurogenesis is propagated (Prada et al., 1991). These differences in the neurogenesis pattern between the DGCs and the other retinal cells can be interpreted as a result of a different regulation of the leaving of the cell cycle.

\section{Morphogenesis}

We have shown in the above section of results that DGCs are generated between E3 and E9. During this time, the newly born DGCs are mixed with ventricular cells (cells in cycle) and young neuroblasts of the different retinal cell types, also generated during this time (Prada et al., 1991). The shapes of these cells with which DGC neuroblasts are mixed were morphologically identified in previous studies (Prada et al., 1981, 1987), using the Golgi method to study the structural changes in the retina from E2 to E8.5. The earliest ganglion cell neuroblasts have monopolar or bipolar shapes detached from the ventricular lining but attached to the vitreal lining, with their somata at different levels of the retinal neuroepithelium. In the present study, we reexamined our Golgi preparations used in previous studies plus a new collection of embryos from E7 to E20, searching specifically for early shapes of ganglion cells that were somewhat different from the ones already identified, which could be assigned to early DGC neuroblasts. Particular attention was paid to the peripheral retina, where DGCs are more numerous in the adult. Up to E7, no such neuroblasts were found.

At E7 we found some shapes that differ from the early ganglion cell neuroblasts, only in having a sprouting (arrow in Fig. 5A) of a few short processes leaving the vitreal process at the level of the prospective ipl. These shapes are proposed as DGC neuroblasts, since at subsequent stages of development this sprouting increases and a sequence of transitional forms is found that would explain their transformation into mature DGCs (Fig. $5 B-$ $H$ ). Furthermore, they were more frequently stained in the peripheral than in the central retina, in agreement with their distribution in the adult.

At E8, clear DGC neuroblasts were found (Fig. $5 B-E$ ). At this stage, ganglion and amacrine cells in the central retina have attained their respective layers, and the ipl has inner and outer limits. DGC neuroblasts are already located among amacrine cells (Fig. 5C), showing an axon (curved arrow) that enters the optic nerve layer through the gcl. The portion of cell that traverses the ipl is thick, and the sprouting around it is more prominent than at E7 (Fig. 5D,E). In some neuroblasts found at this stage, the axon leaves laterally from the thickness of the cell, at the level either of the ipl or of the gcl (Fig. $5 E$ ). These neuroblasts may be the precursors of those that at later stages show the axon leaving from a dendrite, as the shown in Figure $5 I$, found in an E10 retina. The soma of the presumptive early DGC neuroblasts can be rounded (Fig. $5 C$ ) or elongated, and in this case it presents a ventricular process variable in length (Fig. $5 A, B, F$ ). At E9 the youngest DGC neuroblasts (Fig. $5 F, G$ ) have longer processes leaving from the portion of the cell that traverses the ipl. Thus, the initial sprouting seems to mark the position from which the dendritic tree of the cell starts to emerge, and probably also the position of the soma. More mature cells found at this stage in the $\mathrm{C}$ and DP sectors already have a dendritic tree of considerable size (Fig. $5 H$ ), although this is not yet confined to the outer limit of the ipl, as it is in most adult DGCs. At E10 and E11, the soma shapes are very similar to those of the adult, the dendritic tree is already confined to the outer limit of the ipl in the majority of cells, and the soma size is approximately twice or three times that of the amacrine cells (compare, in Fig. 5I, the DGC with the amacrine cell). Two populations of DGCs can be distinguished at these stages, from 

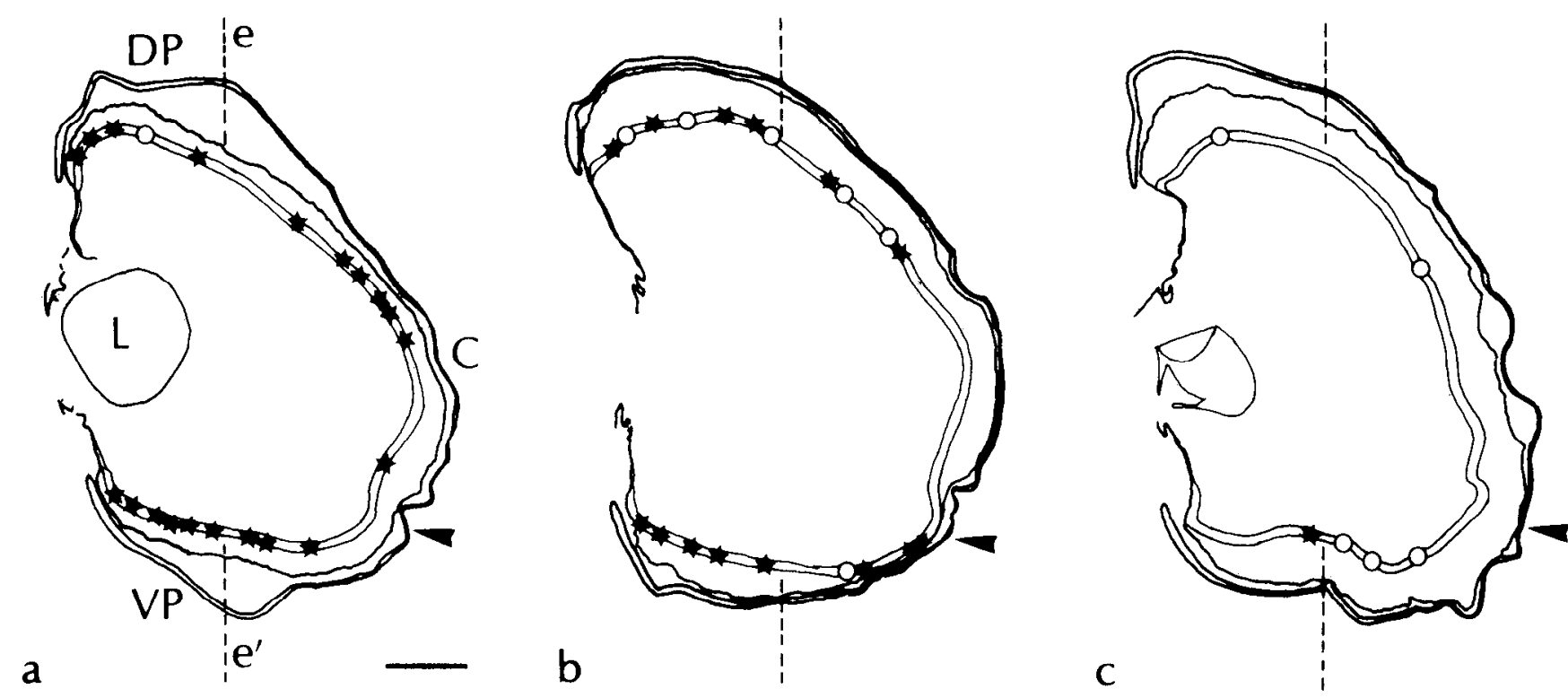

Figure 4. Camera lucida drawings of section autoradiographs of retinas injected at E4 (a), E6 (b), and E7 (c), in which the actual position of all DGCs found, labeled (asterisks) and unlabeled (open circles), is indicated. The equatorial line (ee') indicates the limit between the different retinal sectors considered in this study. These sectors are from dorsal to ventral: $D P$, dorsoperipheral; $C$, central; $V P$, ventroperipheral. $L$, Lens. $A r r o w h e a d s$ indicate the arbitrary limit between the dorsal and ventral retina, determined on the basis of a horizontal plane passing through the dorsal tip of the optic nerve head, and perpendicular to ee', which separates the dorsal and ventral areas of the retina (see Prada et al., 1991, for further explanation). Due to the eccentric position of the optic nerve head (in the ventrotemporal quadrant), the dorsal retina is larger than the ventral. Scale bar, $1.5 \mathrm{~mm}$.

the point of view of the leaving of the axon. In somc cclls, the axon leaves directly from the soma (Fig. $5 J$ ), while in others it does so from a dendrite (Fig. 5I). Both populations were also found in adult retinas (Prada et al., 1989). From these stages onward, little if any modification of the shape can be found.

\section{Discussion}

\section{Neurogenesis}

In the avian retina, DGCs are characterized by unusually large cell bodies (Dogiel, 1888), ranging from 15 to $30 \mu \mathrm{m}$ in the pigeon (Karten et al., 1977; Fite et al., 1981) and from 18 to 42 $\mu \mathrm{m}$ in the chick (Reiner et al., 1979; Prada et al., 1989); this is twice to four times the size of amacrine cell bodies with which they are mixed in the inner row of the inl. Another characteristic is their distribution; studies using retrograde labeling techniques (e.g., La Vail and La Vail, 1974; Reiner et al., 1979; Fite et al., 1981; Hayes and Holden, 1983; Britto et al., 1988) have shown that DGCs are irregularly and widely spaced, and are more frequent in the peripheral than in the central retina. The cells evaluated in our autoradiographs (Fig. 2) have, therefore, the size, location, and distribution shown for DGCs. In addition, the scanning by interferential contrast optics of transverse sections of chick retina, stained by the method selective for DGCs of Goldberg and Frank (1979), as shown by Prada et al. (1989), reveals that only the soma of the largest DGCs protrudes into the ipl (unpublished observations, although Figs. 6 and 7 in Prada et al., 1989, show this). Figure 2 shows how some cells $(b, f, g)$ protrude clearly into the ipl, another strong indication that the cells we evaluated are DGCs. Furthermore, the intense thionin staining of their cytoplasm is in agreement with the very large content of rough endoplasmic reticulum found in DGCs by Prada et al. (1989).

The size of the cells evaluated, more than $18 \mu \mathrm{m}$ in diameter, rules out the possibility that we have counted large amacrine cell bodies of those located in the same row of DGCs. The largest amacrine cells so located, as found in pigeon, are (1) those known as association amacrine cells, which are $13 \mu \mathrm{m}$ in diameter (Mariani, 1982), and (2) catecholaminergic amacrine cells, which are also $13 \mu \mathrm{m}$ in diameter (Britto et al., 1988; Keyser et al., 1990). Their size is clearly below that of the cells evaluated in our work, and coincidentally in both cases the authors recognized that they are below the size of DGCs. In the chick, there are no amacrine cells of $18-92 \mu \mathrm{m}$ in diameter in the same row of DGCs, as can be shown by interferential contrast of retinal sections stained by the method of S. Goldberg and B. Frank (unpublished observations). This is in agreement with the results of Ramón y Cajal (1933).

It is possible that in our evaluations we have not sampled the entire population of DGCs. We have counted only DGCs of large size (compare the enormous size of DGCs in relation to that of ganglion and photorcceptor cells in Fig. $2 h$ ) and wclldelimited nucleus. These large DGCs have been shown to project exclusively upon the accessory optic system in avians ( $\mathrm{La}$ Vail and La Vail, 1974; Reiner et al., 1979; Fite et al., 1981). On the other hand, a population of medium-size DGCs (10-15 $\mu \mathrm{m})$ was described as projecting to the optic tectum in the chick by Crandall et al. (1977) and Heaton et al. (1979), although the existence of this population has been questioned by other investigators (see the discussion in Reiner et al., 1979, and Fite et al., 1981). This last population may exist, but our counts did not include it. However, large DGCs would be the majority in the DGC population, since there are 9000 per retina (mean between close estimation of Reiner et al., 1979, and Prada et al., 1989) versus 2000 medium-size DGCs per retina found by Heaton et al. (1979). The pattern of neurogenesis of the majority of DGCs was determined, however.

Our results show differences in the spatial and temporal patterns of neurogenesis between DGCs and the other retinal cell 

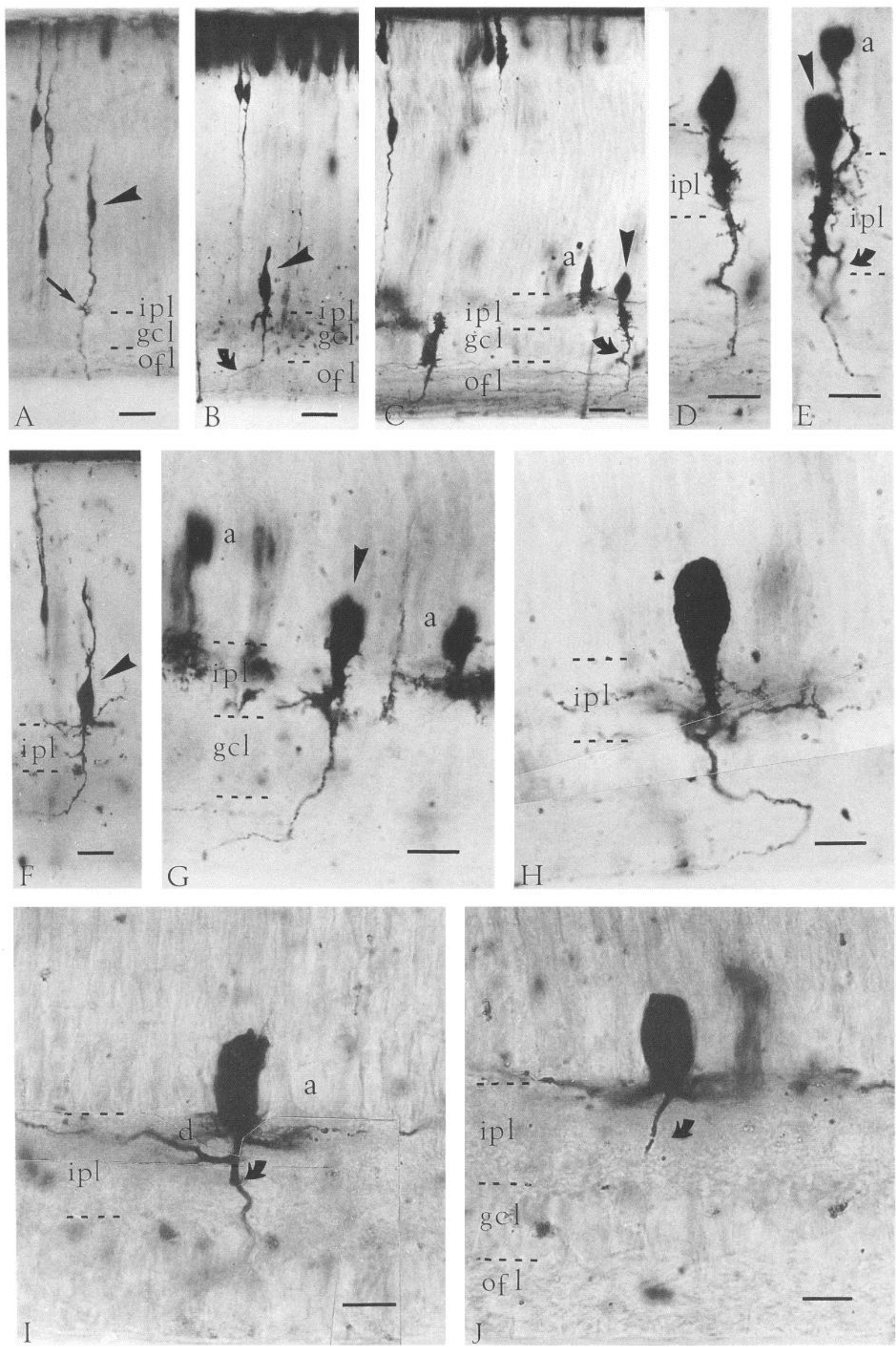
types. The main differences are the following. (1) DGCs start and stop leaving the cycle throughout the entire retina, while the other retinal cell types start in a central area of the dorsotemporal quadrant and end in the VP retina (Prada et al., 1991). (2) Born DGCs are not distributed in gradients during the period of neurogenesis, whilc the other retinal cell types appear in central-to-peripheral, dorsal-to-ventral, and temporal-to-nasal gradients of born cells. (3) The rate of production of DGCs in the dorsal retina is fairly constant with the stage (Fig. 3, C profile), while that of the other retinal cell types changes, being highest during E6 in the dorsocentral sector (see Fig. 9 in Prada et al., 1991). We think that these results taken together point to differences between DGCs and the other retinal cell types in the regulation of the neurogenesis. DGCs may not follow the same general instructions to leave the cycle as the other retinal cell types. Therefore, future investigations of the mechanism(s) of regulation of retinal neurogenesis in the chick would have to examine several questions that arise from our studies: (1) why a certain number of cells of each class is produced in each retinal sector at each stage of neurogenesis, and why such a small number of DGCs is produced; (2) why all cell types with the exception of DGCs start to be produccd in a dctcrmined area of the retina and express spatial gradients of born cells during the neurogenesis period; (3) why the density of DGCs is higher in the peripheral than in the central retina, which is contrary to the behavior of ganglion cells.

As shown in Table 2, small percentages of DGCs start to leave the cycle throughout the retina at E3. A very small number could leave the cycle at E2, because a few unlabeled cells (not considered in our evaluation) were found in retinas labeled at E3. On the other hand, ganglion cells are the first cells in the retina to start leaving the cycle, in a central area of the dorsotemporal quadrant, at E2 (Prada et al., 1991); the peripheral retina does not start to produce ganglion cells until E4. Thus, in the central retina, DGCs start to leave the cycle practically at the same time as ganglion cells, but in the peripheral retina DGCs start to leave the cycle earlier than ganglion cells. Therefore, DGCs are not thc last population of ganglion cells to leave the cycle, and a late neurogenesis cannot be used to explain their "abnormal" position.

DGCs share with the ganglion cells the characteristic of having an axon. However, the axons of DGCs, as already said, project predominantly, if not exclusively, upon extratectal sites in avians (Karten et al., 1977; Fite et al., 1981; Yang et al., 1989), while the axons of ganglion cells project mainly to the optic tectum. Consequently, it has been proposed that DGCs are involved in neural circuits mediating oculomotor reflexes and visuomotor behavior (Fite et al., 1981), this being a different function from that of ganglion cells. In connection with this, we have recently found (Prada et al., 1989) ultrastructural characteristics in DGC's, not shown by ganglion cells, that indicate functional differences, such as the enormous content of rough cndoplasmic reticulum, or the complex synapses (a chemical synapse and an electrical one) received in the initial portion of the axon. These structural and functional differences between DGCs and ganglion cells, together with the characteristic spatial and temporal patterns of the DGC neurogenesis described in the present study, make these cells candidates to be considered a class apart from ganglion cells. Their position around amacrine cells could not be so "abnormal" as has been supposed to date, nor is it a consequence of a late neurogenesis; rather, it seems to be specifically determined during early development.

\section{Morphogenesis}

DGCs start to leave the cycle at E3. However, until E7, no shapes of DGC neuroblasts different from those of ganglion cells were found. There are two possibilities: either both populations of cells have shapes identical to those of the earliest neuroblasts, or the Golgi staining does not specifically stain the earliest DGC neuroblasts. In view of the large number of successfully stained early stage retinas studied, and since the Golgi method in our hands stained even the earliest neuroblasts of the other retinal cell types in the chick (Prada et al., 1981, 1987), the second possibility can be excluded. However, the shapes tentatively identified at $\mathrm{E} 7$ as DGC neuroblasts (Fig. $5 \mathrm{~A}$ ) differ from those of the earliest ganglion cells (see Prada et al., 1981) only in having a sprouting that leaves the vitreal process at the level of the prospective ipl. This is a strong indication that DGCs adopt shapes identical to those of ganglion cells immediately after leaving the cycle, both populations of neuroblasts being indistinguishable until DGC neuroblasts emit the sprouting that characterizes them. Therefore, DGCs would leave the cycle while having interphasic shapes attached to both limiting membranes, detaching them from the ventricular lining as ganglion cells do (Hinds and Hinds, 1974; Prada et al., 1981). Nevertheless, this early sequence of events in DGC morphogenesis can only be demonstrated by means of specific DGC markers of early expression. To our knowledge, no specific marker for these neuroblasts has been found.

At E8, we have unequivocally identified a large number of DGC neuroblasts like that shown in Figure 5, $B$ and $C$. At this stage, the ipl has inner and outer limits in the central retina (Fig. 5C). Ganglion and amacrine neuroblasts have migrated and attained their respective laycrs, and both types of cells have

\footnotetext{
Figure 5. Examples of Golgi-stained DGC neuroblasts, arranged in order of the developmental stage, to show a possible sequence of morphogenesis. In each micrograph, the ventricular (outer) surfaces are toward the top and the vitreal (inner) surfaces are toward the bottom; arrowheads point to the soma, and the curved arrows, to the axon. ipl, inner plexiform layer; gcl, ganglion cell layer; ofl, optic fiber layer. $A$, Presumptive migrating DGC neuroblast of a retina from an E7 embryo. The arrow points to an incipient sprouting that appears in the prospective ipl before the axon had begun to grow. $B$, and $C$, Neuroblasts of retinas from $\mathrm{E} 8$ embryos; their cell bodies are already located among amacrine cells $(a$ in $C)$ and bear an axon that traverses the gcl. Note that in both examples the portion of cell that traverses the ipl has thickened and the sprouting has increased; however, they differ in the shape of the soma. $D$ is an enlargement of cell in $C$, to show in more detail the portion of the cell that traverses the ipl. $E$, Neuroblast of a retina from an E8 embryo that differs from the one shown in $D$ in having an axon leaving laterally the portion of the cell that traverses the ipl; $a$, amacrine cell. $F-H$, Neuroblasts of retinas from $\mathrm{E} 9$ embryos. Note that the neuroblast in $F$ could represent a more advanced stage of transformation of shapcs like those shown in $A$ and $B$, since they all have a ventricular process. In $G$, the two amacrine cells $(a)$ show that the ganglion cell between them is a displaced one. $I$, DGC of a retina from an E10 embryo with the appearance of a mature cell whose axon leaves from a dendrite $(d)$, unlike other DGCs of the same stage that have the axon leaving directly from the soma. Observe the large size of the DGC soma in relation to that of the amacrine cell $(a)$ on the right, just visible by contrast. $J$, Example of a DGC of a retina from an E11 embryo whose axon leaves the soma. In all illustrations, the limits between layers were checked by light contrast, as shown in $I$ and $J$. Scale bars, $30 \mu \mathrm{m}$.
} 
started to grow the dendritic tree (Prada et al., 1987). As shown in Figure 5, $C$ and $D$, some neuroblasts are located among amacrine cells, which show processes horizontally extended at the outer limit of the ipl, a pronounced sprouting at the level of the ipl, and an axon that joins with the axons of ganglion cells at the optic fiber layer, offering little doubt that they are DGC neuroblasts. On the other hand, ganglion cell neuroblasts do not emit processes at the level of the ipl during migration, either in the chick (Prada et al., 1981) or in the rat (Hinds and Hinds, 1974), and therefore this excludes the possibility that the neuroblasts identified at E8 as DGCs are migrating ganglion cell neuroblasts. From E8 onward, DGC shapes are clearly distinguishable from any others in the retina.

\section{References}

Boycott BB, Dowling JE (1969) Organization of the primate retina: light microscopy. Philos Trans R Soc Lond [Biol] 255:109-184.

Britto LRG, Keyser KT, Hamassaki DE, Karten HJ (1988) Catecholaminergic subpopulation of retinal displaced ganglion cells projects to the accessory optic nucleus in the pigeon (Columba livia). J Comp Neurol 269:109-117.

Buhl EH, Dann JF (1988) Morphological diversity of displaced retinal ganglion cells in the rat: a Lucifer yellow study. J Comp Neurol 269: 210-218.

Bunt AH, Minckler DS (1977) Displaced ganglion cells in the retina of the monkey. Invest Ophthalmol Vis Sci 16:95-98.

Colonnier M (1964) The tangential organization of the visual cortex. $\mathrm{J}$ Anat 98:327-334.

Crandall JE, Heaton MB, Brownell WE (1977) Tectal projection of displaced ganglion cells in avian retina. Invest Ophthalmol Vis Sci $16: 774-776$

Dogiel AS (1888) Über das verhalten der nervösen elemente in der retina der ganoiden, reptilien, vögel und sängetiere. Anat Anz 3:133143.

Dräger UC, Olsen JF (1980) Origins of crossed and uncrossed retinal projections in pigmented and albino mice. J Comp Neurol 191:383412 .

Dräger UC, Olsen JF (1981) Ganglion cell distribution in the retina of the mouse. Invest Ophthalmol Vis Sci 20:285-293.

Fite KV, Brecha N, Karten HJ, Hunt SP (1981) Displaced ganglion cells and the accessory optic system of pigeon. J Comp Neurol 195: 279-288.

Goldberg S, Frank B (1979) Pyridin-silver method for the study of optic axons in retina whole mounts. Stain Technol 54:21-23.

Hamburger V, Hamilton HL (1951) A series of normal stages in the development of the chick embryo. J Morphol 88:48-92.

Hayes BP, Holden AL (1983) The distribution of displaced ganglion cells in the retina of the pigeon. Exp Brain Res 49:181-188.

Heaton MB, Alvarez IM, Crandall JE (1979) The displaced ganglion cell in the avian retina: developmental and comparative considerations. Anat Embryol 155:161-178.
Hinds JW, Hinds PL (1974) Early ganglion cell differentiation in the mouse retina: an electron microscopic analysis utilizing serial sections. Dev Biol 37:381-416.

Kahn AJ (1973) Ganglion cell formation in the chick neural retina. Brain Res 63:285-290.

Kahn AJ (1974) An autoradiographic analysis of the time of appearance of neurons in the developing chick neural retina. Dev Biol 38 $30-40$.

Karten HJ, Fite KV, Brecha N (1977) Specific projection of displaced ganglion cells upon accessory optic system in the pigeon (Columbia livia). Proc Natl Acad Sci USA 74:1753-1756.

Keyser KT, Britto LRG, Woo J-I, Park DH, Joh TH, Karten HJ (1990) Presumptive catecholaminergic ganglion cells in the pigeon retina. Vis Neurosci 4:225-235.

I a Vail JH, La Vail MJ (1974) The retrograde intra-axonal transport of horseradish peroxidase in the chick visual system: a light and electron microscopy study. J Comp Neurol 157:303-358.

Linden R (1987) Displaced ganglion cells in the retina of the rat. J Comp Neurol 258:138-143.

Mariani AP (1982) Association amacrine cells could mediate directional selectivity in pigeon retina. Nature 298:654-655.

Maslim J, Webster M, Stone J (1986) Stages in the structural differentiation of retinal ganglion cells. J Comp Neurol 254:382-402.

Prada C, López-Mascaraque L (1985) Dammar resin prevents the fading of celloidin sections of Golgi impregnated embryonic central nervous system. Mikroskopie 42:146-147.

Prada C, Puelles L, Génis-Gálvez JM (1981) A Golgi study on the early sequence of differentiation of ganglion cells in the chick embryo retina. Anat Embryol 161:305-317.

Prada C, Puelles L, Génis-Gálvez JM, Ramirez G (1987) Two modes of free migration of amacrine cell neuroblasts in the chick retina. Anat Embryol 175:281-287.

Prada C, Puga J, Perez-Mendez L, Lopez R, Ramírez G (1991) Spatial and temporal patterns of neurogenesis in the chick retina. Eur J Neurosci 3:559-569.

Prada FA, Chmielewski CE, Dorado ME, Prada C, Génis-Gálvez JM (1989) Displaced ganglion cells in the chick retina. Neurosci Res 6: 329-339.

Ramón y Cajal S (1933) La rétine des vertébrés. Translated in: The structure of the retina (Thorpe SA, Glickstein M, eds), pp 76-92. Springfield, IL: Thomas, 1972.

Reiner A, Brecha N, Karten HJ (1979) A specific projection of retinal displaced ganglion cells to the nucleus of the basal optic root in the chicken. Neuroscience 4:1679-1688.

Stensaas LJ (1967) The development of hippocampal and dorsolateral pallial regions of the cerebral hemisphere in fetal rabbits. I. Fifteen millimeter stage, spongioblast morphology. J Comp Neurol 129:5970

Yang G, Millar TJ, Morgan IG (1989) Co-lamination of cholinergic amacrine cell and displaced ganglion cell dendrites in the chicken retina. Neurosci Lett 103:151-156. 